\title{
Equality in László Fejes Tóth's triangle bound for hyperbolic surfaces
}

\author{
Christophe Bavard, Károly J. Böröczky*, \\ Borbála Farkas, István Prok, Lluis Vena, Gergely Wintsche
}

November 1, 2021

\begin{abstract}
For $k \geq 7$, we determine the minimal area of a compact hyperbolic surface, and an oriented compact hyperbolic surface that can be tiled by embedded regular triangles of angle $2 \pi / k$. Based on this, all the cases of equality in László Fejes Tóth's triangle bound for hyperbolic surfaces are described.
\end{abstract}

\section{Introduction}

Let $\mathbb{H}^{2}$ be the hyperbolic plane of curvature -1 (see J.G. Ratcliffe [7] for facts and references on hyperbolic geometry, and D.V. Alekseeskij, E.B. Vinberg, A.S. Solodovnikov [1 for an in depth study on discrete groups of isometries). A compact hyperbolic surface $X$ can be obtained as the quotient of $\mathbb{H}^{2}$ by a discrete group $\Gamma$ of isometries that acts fixed point free on $\mathbb{H}^{2}$. For a regular polygon $\Pi$ in $\mathbb{H}^{2}$, we say that $X$ can be tiled by copies of $\Pi$ if there exists an edge to edge tiling of $\mathbb{H}^{2}$ by congruent copies of $\Pi$ that is invariant under $\Gamma$. The equivalence classes of the tiles, edges, and vertices of the tiling in $\mathbb{H}^{2}$ with respect to $\Gamma$ correspond to the tiles, edges, and vertices, respectively, of the tiling on $X$ (as a CW-decomposition of $X$ ). In this case there exists an integer $p \geq 3$ such that the angles of $\Pi$ are $2 \pi / p$. If $\Pi$ is a regular triangle then $p \geq 7$.

In this note, we provide a simple proof for the following statement.

*Supported by OTKA grants 068398 and 75016 , and by the EU Marie Curie TOK project DiscConvGeo and FP7 IEF grant GEOSUMSETS 
Theorem 1.1 For $k \geq 7$, the minimal area of a compact hyperbolic surface that can be tiled by embedded regular triangles of angle $2 \pi / k, k \geq 7$, is $N(k-6) \frac{\pi}{3}$, where $N$ is the minimal positive integer such that $N k$ is divisible by six. If the surface is assumed to be orientable then the minimal area is the same if $k \equiv 2,6,10 \bmod 12$, and twice the previous value otherwise.

Remark It follows by A.L. Edmonds, J.H. Ewing, R.S. Kulkarni [4] that there is a compact hyperbolic surface that can be tiled by $t$ embedded regular triangles of angle $2 \pi / k, k \geq 7$, if and only if $t$ is even, and $3 t$ is divisible by $k$.

We note that Theorem 1.1 is proved in a more general setup, and using a more involved construction in A.L. Edmonds, J.H. Ewing, R.S. Kulkarni [3]. In addition, the case of Theorem 1.1 when $k$ is divisible by six, is proved by C. Bavard [2].

In other words, for $k \geq 7$, let $\Gamma_{k}$ be the isometry group of the edge to edge tiling by regular triangles of angle $2 \pi / 7$. Then Theorem 1.1 yields (using its notation) that the minimal index of a subgroup of $\Gamma_{k}$ that acts fixed point free on $\mathbb{H}^{2}$ is $2 N k$.

Our motivation to consider Theorem 1.1 comes from the theory of packing and covering by equal circular discs on a compact surface $X$ of constant curvature. Let $T$ be a regular triangle of angle $\alpha$ in the universal covering surface, which is $\mathbb{H}^{2}, \mathbb{R}^{2}$, and the unit sphere $S^{2}$ in $\mathbb{R}^{3}$ if the constant curvature is $-1,0$ or 1 , respectively. In addition, let $r$ and $R$ be inradius and circumradius, respectively, of $T$, and let $A(\cdot)$ denote area. According to the triangle bound due to L. Fejes Tóth [6] (see also G. Fejes Tóth, W. Kuperberg [5] for a survey), if $X$ can be covered by $n$ metric balls of radius $R$, then

$$
A(X) \leq n \cdot \frac{2 \pi}{3 \alpha} \cdot A(T) .
$$

In addition, if $X$ contains $n$ pairwise disjoint open embedded balls of radius $r$, then

$$
A(X) \geq n \cdot \frac{2 \pi}{3 \alpha} \cdot A(T) .
$$

If the injectivity radius of $X$ is at least $r$, then $A(X) \geq \frac{2 \pi}{3 \alpha} \cdot A(T)$. In these bounds, equality holds if and only if $\alpha=2 \pi / k$ for some integer $k \geq 3$, and $X$ can be tiled by copies of $T$ using exactly $n$ vertices. To characterize the cases when we have equality in the estimate using the injectivity radius $(n=1)$ is the subject of C. Bavard [2].

To charaterize regular triangulations when the curvature of $X$ is nonnegative, is rather straightforward. If the curvature of $X$ is positive, then $k \in\{3,4,5\}$, as the sum of the angles of $T$ is larger, than $\pi$. There are 
two types of possible $X$. If $X$ is a sphere then the possible regular triangulations are determined by the platonic solids: tetrahedron, octahedron and icosahhedron. If $X$ is a projective plane then the possible regular triangulations are obtained by identifying the opposite faces of the octahedron and the icosahhedron.

Next, there are exactly two types of surfaces of curvature zero; namely, tori and Klein bottles. In these cases $k=6$. For any $n \geq 1$, it is easy to construct a surface of either type with a regular triangulation having $n$ vertices.

Therefore, it remains to find regularly triangulated hyperbolic surfaces, when $k \geq 7$. This is the content of Theorem 1.1 and the following remark.

\section{Some basic properties of hyperbolic sur- faces}

First, we provide some properties of tilings of a compact hyperbolic surface $X$. The Euler characteristic of $X$ is denoted by $\chi(X)$, which is negative, and is always even in the case of oriented surfaces. It follows from the GaußBonnet formula that the area of $X$ is $2 \pi|\chi(X)|$, therefore minimizing the area is equivalent to maximizing the Euler characteristic.

Lemma 2.1 If a compact hyperbolic surface $X$ is tiled by embedded regular triangles of angle $2 \pi / k, k \geq 7$, and the tiling has $n$ vertices (as a $C W$ decomposition), then $n k$ is divisible by six, and

$$
\chi(X)=n \cdot\left(1-\frac{k}{6}\right) .
$$

Proof: Since each vertex of the tiling is incident with exactly $k$ edges, the tiling has $n k / 3$ faces and $n k / 2$ edges. Therefore, we have

$$
\chi(X)=n-\frac{n k}{2}+\frac{n k}{3}=n \cdot\left(1-\frac{k}{6}\right) .
$$

Since $n k$ is divisible by two and three, it is divisible by six. Q.E.D.

After the necessary condition above, we present some ways to construct suitable hyperbolic surfaces. Using the oriented double cover of a nonorientable surface, we have the following.

Lemma 2.2 If a compact non-orientable hyperbolic surface $X$ is tiled by embedded regular triangles of angle $2 \pi / k, k \geq 7$, using $n$ vertices, then there exists a compact oriented hyperbolic surface $\tilde{X}$, which is tiled by embedded regular triangles of angle $2 \pi / k$ using $2 n$ vertices, and $\chi(\tilde{X})=2 \chi(X)$. 
Assume that a compact hyperbolic surface $X$ is tiled by embedded regular triangles of angle $2 \pi / k, k \geq 7$, and the tiling has $n$ vertices. By duality, it is equivalent that $X$ can be tiled by $n$ embedded regular $k$-gons of angle $2 \pi / 3$.

To obtain $X$, one may start with $n$ regular $k$-gons, and identify the suitable pairs out of the total $n k$ edges of the $n$-gons. We describe a combinatorial way to encode pairings that we need in the sequel.

Let $\Pi_{1}, \ldots, \Pi_{n}$ be convex polygons with at least seven vertices such that the their total number of vertices (or edges) is $3 m$ for some even integer $m$. A proper labeling of the vertices of $\Pi_{1}, \ldots, \Pi_{n}$ means an assignment of a label from $\{1, \ldots, m\}$ to each vertex with the following properties.

(i) Any $i \in\{1, \ldots, m\}$ is the label of exactly three of the vertices.

(ii) For any non-empty proper subset of $\Pi_{1}, \ldots, \Pi_{n}$ there exists a label $i \in$ $\{1, \ldots, m\}$ that occurs once or twice as the label of some vertex of a convex polygon in the subset.

(iii) If a $\Pi_{l}$ has two consecutive vertices with label $i \in\{1, \ldots, m\}$, then $\Pi_{l}$ has three consecutive vertices with label $i$. They determine two consecutive edges of $\Pi_{l}$, which are called proper pairs.

(iv) If $i \neq j$ are the labels of the end points of an edge $e$ of some $\Pi_{l}$, then there exists exactly one more edge among the all together $3 m$ edges, whose endpoints are labeled $i$ and $j$. This edge is called the proper pair of $e$.

(v) It never occurs that for some $i \neq j$, the two neighbours of a vertex with label $i$ are both labeled $j$.

Given a proper labeling, we give a orientation to each edge. For an edge $e$ of some $\Pi_{l}$, whose end points are labeled $i$ and $j$, we orient the edge $e$ according to the natural ordering of the labels of its vertices if $i \neq j$, and according to the positive orientation of $\Pi_{l}$ if $i=j$. We say that the orientation of $e$ is positive if it coincides with the orientation induced by the positive orientation of $\Pi_{l}$, and the orientation of $e$ is negative otherwise. We say that the proper labeling is oriented, if for any proper pair $e$ and $f$ of edges, the orientations of $e$ and $f$ are opposite.

For $k \geq 7$, let us assume that $\Pi_{1}, \ldots, \Pi_{n}$ are regular $k$-gons with angle $2 \pi / k$, and we have have a proper labeling of the vertices. Then identifying the proper pairs of edges according their orientations, we obtain a (connected) compact hyperbolic surface $X$, which is oriented if the proper labeling is oriented. 


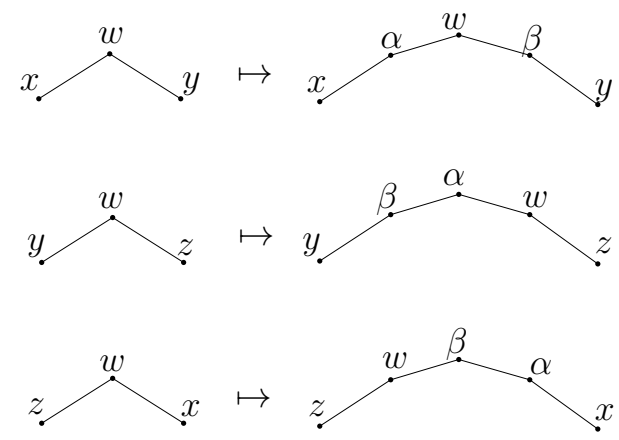

Figure 1:

From a given proper labeling of $n$ polygons, we will construct a proper labeling of $n$ polygons with a higher number of vertices using the operations (a), (b) and (c) below.

A polygonal path $a_{1} \ldots a_{m}, m \geq 2$, is the union of the segments with end points $a_{i}$ and $a_{i+1}, i=1, \ldots, m-1$, where $a_{1}, \ldots, a_{m}$ are different. When we replace a polygonal path $a_{1} \ldots a_{m}$ contained in the boundary of a convex polygon $\Pi$ with the polygonal path $b_{1} \ldots b_{l}$ with $a_{1}=b_{1}$ and $a_{m}=b_{l}$, we always do it in a way to obtain a new convex polygon $\Pi^{\prime}$, where $b_{1}, \ldots, b_{l}$ are vertices of $\Pi^{\prime}$.

Let us assume that we have a proper labeling of vertices of the convex polygons $\Pi_{1}, \ldots, \Pi_{n}$ using labels from $\{1, \ldots, m\}$. When replacing a polygonal path with a new one, we only list the corresponding labels.

(a) at $w$ Assume that for $\{x, y, z, w\} \subset\{1, \ldots, m\}$, the union of the boundaries of $\Pi_{1}, \ldots, \Pi_{n}$ contains polygonal paths with labels $x w y, y w z$ and $z y x$. Then for $\alpha=m+1$ and $\beta=m+2$, we replace (see Figure 1)

$$
\begin{array}{lll}
x w y & \text { by } & x \alpha w \beta y \\
y w z & \text { by } & y \beta \alpha w z \\
z w x & \text { by } & z w \beta \alpha x .
\end{array}
$$

(b) at $w$ Assume that for $\{x, y, z, w\} \subset\{1, \ldots, m\}$, the union of the boundaries of $\Pi_{1}, \ldots, \Pi_{n}$ contains polygonal paths with labels $x w y, y w z$ and $z y x$. Then for $\alpha=m+1, \beta=m+2, \gamma=m+3$ and $\delta=m+4$, we replace (see Figure 2)

$$
\text { xwy by } \quad x \alpha \gamma w \delta \beta y
$$




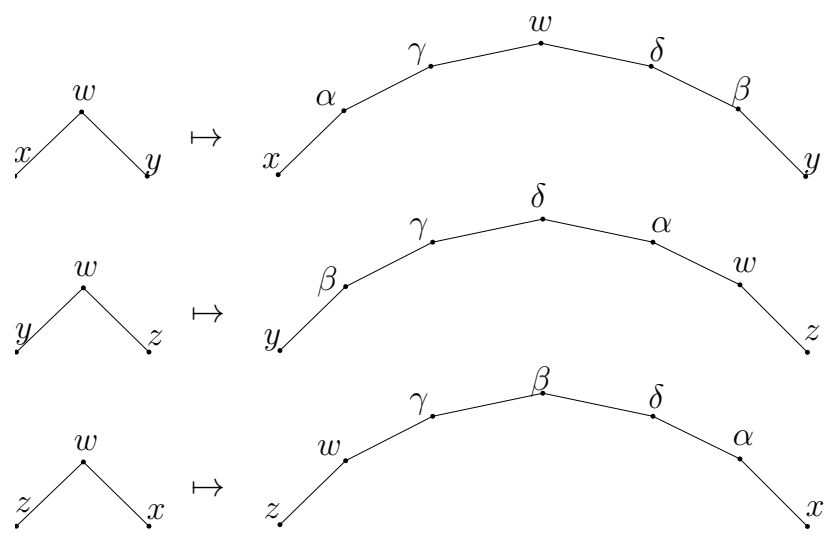

Figure 2:

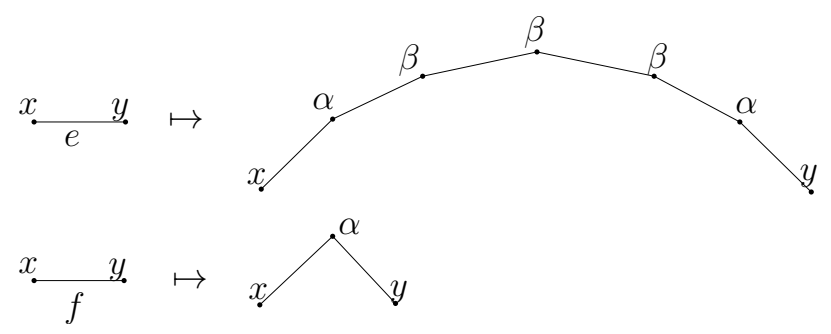

Figure 3:

$$
\begin{array}{lll}
y w z & \text { by } & y \beta \gamma \delta \alpha w z \\
z w x & \text { by } & z w \gamma \beta \delta \alpha x .
\end{array}
$$

(c) at $x, y$ Assume the endpoints of the proper pair of edges $e$ and $f$ are labeled $x \neq y$. For $\alpha=m+1$ and $\beta=m+2$, we replace (see Figure 3)

$e$ by the polygonal path $x \alpha \beta \beta \beta \alpha y$

$f$ by the polygonal path $x \alpha y$.

We observe that all operations (a), (b) and (c) produce a new proper labeling. In addition, if the proper labeling of the vertices of $\Pi_{1}, \ldots, \Pi_{n}$ is oriented, then the new proper labeling constructed in (b) is also oriented. 


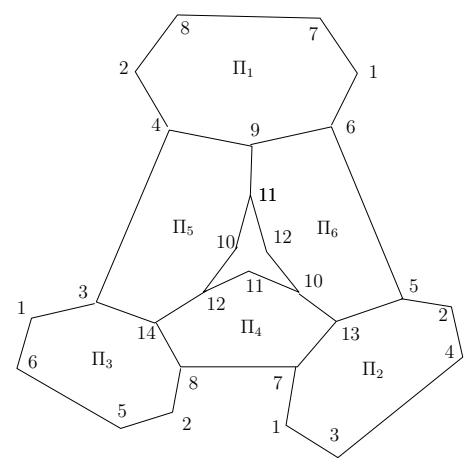

Figure 4:

\section{Proof of Theorem 1.1}

For $k \geq 7$, let $N(k)$ be the smallest positive integer such that $N(k) \cdot k$ is divisible by six. It follows by Lemma 2.1 that if a compact hyperbolic surface $X$ is tiled by embedded regular triangles of angle $2 \pi / k, k \geq 7$, then

$$
\chi(X)=l \cdot N(k) \cdot\left(1-\frac{k}{6}\right) \text { for a positive integer } l .
$$

In addition, let $X_{k}\left(\widetilde{X}_{k}\right)$ be a hyperbolic surface (oriented hyperbolic surface) of smallest area that can be tiled by embedded regular triangles of angle $2 \pi / k$. Theorem 1.1 is equivalent proving that

$$
\text { there exists a proper labeling of } N(k) k \text {-gons. }
$$

It follows by Lemma 2.2 and (1) that

$$
\text { if } \chi\left(X_{k}\right)=N(k)\left(1-\frac{k}{6}\right) \text { and } \chi\left(X_{k}\right) \text { is odd then } \chi\left(\widetilde{X}_{k}\right)=2 \chi\left(X_{k}\right) \text {. }
$$

We construct $X_{k}$ and $\widetilde{X}_{k}$, depending on the remainder of $k$ modulo 12 , and providing a proper labeling of the vertices of $N(k) k$-gons. We only provide the $N(k)$ lists of labels according to positive orientation of the $k$-gons.

Case $1 \quad k \equiv 1,5,7,11 \bmod 12$

In these cases, we have $N(k)=6$. If $k=7$, then the following is a proper labeling (see Figure 4):

$$
\begin{array}{ll}
\Pi_{1}: & 1,6,9,4,2,8,7 \\
\Pi_{2}: & 1,7,13,5,2,4,3
\end{array}
$$




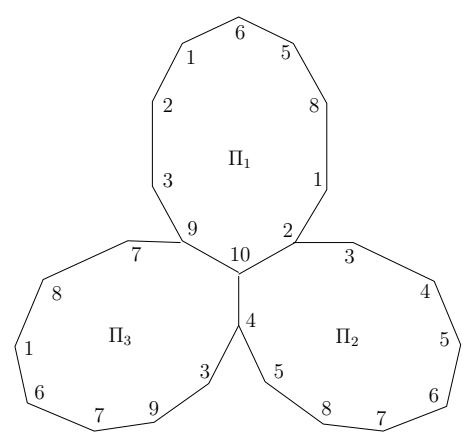

Figure 5:

$$
\begin{array}{ll}
\Pi_{3}: & 1,3,14,8,2,5,6 \\
\Pi_{4}: & 7,8,14,12,11,10,13 \\
\Pi_{5}: & 3,4,9,11,10,12,14 \\
\Pi_{6}: & 9,6,5,13,10,12,11
\end{array}
$$

To prove (2) for $k=11$, we apply operation (b) at 1 (to alter $\Pi_{1}, \Pi_{2}$ and $\Pi_{3}$ ), and then again (b) at 10 (to alter $\Pi_{4}, \Pi_{5}$ and $\Pi_{6}$ ). This way we obtain a proper labeling of some six 11-gons. After this, we apply (a) at 1 and 10 to obtain a proper labeling of some six 13-gons. Continuing this way, applying alternately operation (b) at 1 and 10, and operation (a) at 1 and 10, we obtain a proper labeling of some six $k$-gons for any $k$ in Case 1.

For these $k$, we have $\chi\left(X_{k}\right)=6-k$ by Lemma 2.1, which is odd. Therefore, $\chi\left(\tilde{X}_{k}\right)=2 \chi\left(X_{k}\right)$ by $(3)$.

Case $2 k \equiv 2,10 \bmod 12$

In these cases, we have $N(k)=3$. If $k=10$, then the following is an oriented proper labeling (see Figure 5):

$$
\begin{array}{ll}
\Pi_{1}: & 10,9,3,2,1,6,5,8,1,2 \\
\Pi_{2}: & 10,2,3,4,5,6,7,8,5,4 \\
\Pi_{3}: & 10,4,3,9,7,6,1,8,7,9
\end{array}
$$

To prove (2) for $k=14$, we apply operation (b) at 10 . To prove (2) for $k=22$, we apply operation (b) at 10 twice. In particular, using operation (b) at 10, we obtain a proper labeling of some three $k$-gons for any $k$ in Case 2 .

Since each one of the proper labelings presented in Case 2 is oriented, the $X_{k}$ constructed this way is also oriented. 


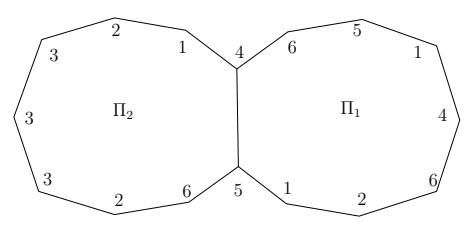

Figure 6:

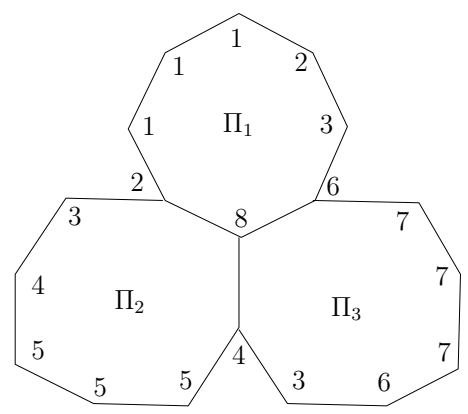

Figure 7:

Case $3 \quad k \equiv 3,9 \bmod 12$

In these cases, we have $N(k)=2$. If $k=9$, then the following is a proper labeling (see Figure 6):

$$
\begin{array}{ll}
\Pi_{1}: & 4,6,5,1,4,6,2,1,5 \\
\Pi_{2}: & 4,5,6,2,3,3,3,2,1 .
\end{array}
$$

To prove (2) for $k=15$, we apply operation (c) at 4,5. In particular, using operation (b) at 4,5 , we obtain a proper labeling of some pair of $k$-gons for any $k$ in Case 3 .

For these $k$, we have $\chi\left(X_{k}\right)=2-\frac{k}{3}$ by Lemma 2.1. which is odd as $\frac{k}{3}$ is odd. Therefore, $\chi\left(\widetilde{X}_{k}\right)=2 \chi\left(X_{k}\right)$ by $(3)$.

Case $4 k \equiv 4,8 \bmod 12$

In these cases, we have $N(k)=3$. If $k=8$, then the following is a proper labeling (see Figure 7):

$$
\begin{array}{ll}
\Pi_{1}: & 8,2,1,1,1,2,3,6 \\
\Pi_{2}: & 8,4,5,5,5,4,3,2 \\
\Pi_{3}: & 8,6,7,7,7,6,3,4 .
\end{array}
$$

To prove (2) for $k=16$, we apply operation (b) at 8 twice. To prove (2) for 


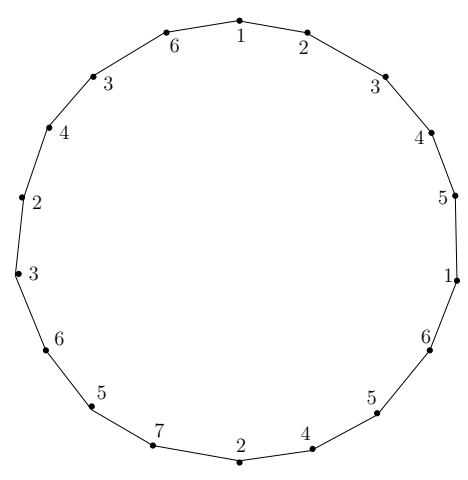

Figure 8:

$k=20$, we apply operation (b) at 8. In particular, using operation (b) at 8 , we obtain a proper labeling of some three $k$-gons for any $k$ in Case 4.

For these $k$, we have $\chi\left(X_{k}\right)=3-\frac{k}{2}$ by Lemma 2.1, which is odd as $\frac{k}{2}$ is even. Therefore, $\chi\left(\widetilde{X}_{k}\right)=2 \chi\left(X_{k}\right)$ by $(3)$.

Case $5 \quad k \equiv 6 \bmod 12$

In this case, we have $N(k)=1$. If $k=18$, then the following is an oriented proper labeling (see Figure 8):

$$
\Pi_{1}: \quad 1,2,3,4,5,1,6,5,4,2,7,5,6,3,2,4,3,6 .
$$

To prove (2) for $k=30$, we apply operation (b) at 1 three times. In particular, using operation (b) at 1, we obtain an oriented proper labeling of some $k$-gon for any $k$ in Case 5 . Therefore, $\chi\left(\widetilde{X}_{k}\right)=\chi\left(X_{k}\right)=1-\frac{k}{6}$ in this case.

Case $6 \quad k \equiv 0 \bmod 12$

In this case, we have $N(k)=1$. If $k=12$, then the following is a proper labeling (see Figure 9):

$$
\Pi_{1}: \quad 1,2,3,4,1,3,2,4,3,1,2,4 .
$$

To prove (2) for $k=24$, we apply operation (b) at 1 three times. In particular, using operation (b) at 1 , we obtain a proper labeling of some $k$-gon for any $k$ in Case 6 .

For these $k$, we have $\chi\left(X_{k}\right)=1-\frac{k}{6}$ by Lemma 2.1. which is odd as $\frac{k}{6}$ is even. Therefore, $\chi\left(\widetilde{X}_{k}\right)=2 \chi\left(X_{k}\right)$ by (3). Q.E.D.

Remark If $k \geq 7$ is divisible by six, then there is a graph theoretic way to produce a proper labeling of a $k$-gon. Let $G$ be a connected graph on 


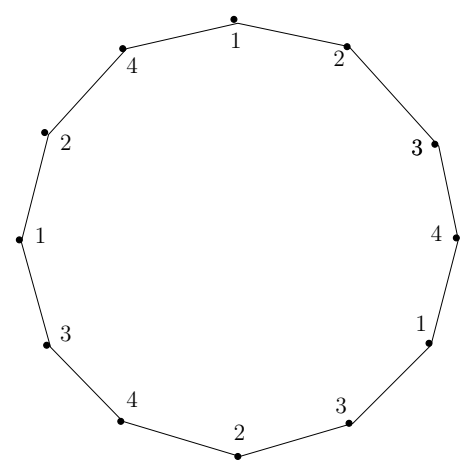

Figure 9:

$\{1, \ldots, m\}$ for $m=k / 3$ such that each vertex is of degree three. We call a closed path on the edges of $G$ a double Hamiltonian path if each edge is traveled exactly twice, and consecutive edges in the path are always different. Then a proper labeling of the vertices of the $k$-gon where no pair of consecutive vertices have the same label, is equivalent to a double Hamiltonian path. The proper labeling is oriented if and only if each edge is traveled in both directions in the double Hamiltonian path.

\section{References}

[1] D.V. Alekseeskij, E.B. Vinberg, A.S. Solodovnikov: Geometry of spaces of constant curvature. Encycl. Math. Sci. 29, Springer-Verlag, 1993, 1138.

[2] C. Bavard: Disques extrémaux et surfaces modulaires. Ann. Fac. Sci. Toulouse Math., 5 (1996), 191-202.

[3] A.L. Edmonds, J.H. Ewing, R.S. Kulkarni: Regular tessellations of surfaces and (p, q, 2)-triangle groups. Ann. Math., 116 (1982), 113-132.

[4] A.L. Edmonds, J.H. Ewing, R.S. Kulkarni: Torsion Free Subgroups of Fuchsian Groups and Tessalations of Surfaces. Inventiones Mathematicae, 69 (1982), 331-346.

[5] G. Fejes Tóth, W. Kuperberg: Packing and covering with convex sets, In: Handbook of Convex Geometry, P. Gruber and J. Wills (eds.), North-Holland 1993, 799-860.

[6] L. Fejes Tóth: Regular Figures. Pergamon Press, 1964. 
[7] J.G. Ratcliffe: Foundations of hyperbolic manifolds. Springer, 1994.

Christophe Bavard, Christophe.Bavard@math.u-bordeaux.fr Institut de Mathématiques, Université Bordeaux 1

Károly J. Böröczky, carlos@renyi.hu Alfréd Rényi Institute of Mathematics, and Universitat Politècnica de Catalunya, Barcelona Tech, and Department of Geometry, Roland Eötvös University

Borbála Farkas, farkas_borka@yahoo.co.uk Department of Geometry, Technical University of Budapest

István Prok,prok@math.bme.hu

Department of Geometry, Technical University of Budapest

Lluis Vena,lluis.vena@utoronto.ca

Department of Mathematics, University of Toronto

Gergely Wintsche, wgerg@ludens.elte.hu

Department of Didactics, Roland Eötvös University 